\title{
Measurement of the Hadronic Cross-Section for the Scattering of Two Virtual Photons at LEP
}

\section{Mariusz Przybycień*}

University of Mining and Metallurgy, Cracow, Poland

\begin{abstract}
The interactions of virtual photons are investigated based on data taken by three LEP experiments ALEPH, L3 and OPAL, at $e^{+} e^{-}$centre of mass energies $\sqrt{s_{e e}}=$ 189-209 GeV, for two photon invariant mass $W>5 \mathrm{GeV}$, using the reaction $e^{+} e^{-} \rightarrow$ $e^{+} e^{-}$hadrons. The measured cross sections are compared to the predictions of the Quark Parton Model (QPM), to the LO QCD Monte Carlo models PHOJET, TWOGAM and PYTHIA, to the NLO prediction for the reaction $e^{+} e^{-} \rightarrow e^{+} e^{-} q \bar{q}$, and to BFKL calculations. The $\mathrm{LO} \mathrm{MC}$ models, $\mathrm{NLO} e^{+} e^{-} \rightarrow e^{+} e^{-} q \bar{q}$ and $\mathrm{QPM}$ describe the data reasonably well, whereas the cross section predicted by a LO BFKL calculation is too large.
\end{abstract}

\section{Introduction}

The classical way to investigate the structure of the photon at $\mathrm{e}^{+} \mathrm{e}^{-}$colliders is the measurement of the process, $e\left(p_{1}\right) e\left(p_{2}\right) \rightarrow e\left(p_{1}^{\prime}\right) e\left(p_{2}^{\prime}\right)$ hadrons, proceeding via the exchange of two photons, which can be either quasi-real, $\gamma$, or virtual $\gamma^{\star}$. The terms in brackets represent the four-vectors of the particles as shown in Fig. 1‥1. In the analyses presented here both final state electrons are observed, which means they must be scattered at sufficiently large polar angles $\theta_{i}(i=1,2$ denotes quantities which are connected with the upper/lower vertex in Fig. ii i $)$ and consequently both radiated photons, which take part in the hard scattering process, are highly virtual. For the detailed discussion of the theory of two photon interactions see e.g. [1] The detailed description of the measurements presented in this

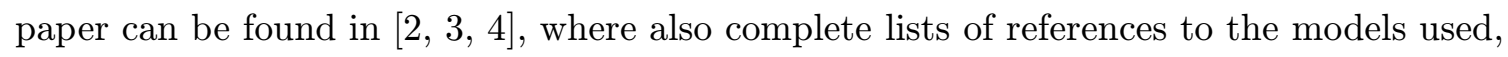
are given. In the following only main points of the analyses and the results are presented.

The kinematical variables $Q_{i}^{2}, y_{i}$ and $x_{i}$ are obtained from the four-vectors of the tagged electrons and the hadronic final state via: $Q_{i}^{2}=2 E_{\mathrm{b}} E_{i}\left(1-\cos \theta_{i}\right), y_{i}=1-E_{i} / E_{\mathrm{b}} \cos ^{2}\left(\theta_{i} / 2\right)$, $x_{i}=Q_{i}^{2} /\left(Q_{1}^{2}+Q_{2}^{2}+W^{2}\right)$, where $E_{\mathrm{b}}$ is the beam energy, and the electron mass has been neglected. The $\mathrm{e}^{+} \mathrm{e}^{-}$centre-of-mass energy squared is given by $s_{\text {ee }}=\left(p_{1}+p_{2}\right)^{2}$ and the hadronic invariant mass squared by $W^{2}=\left(q_{1}+q_{2}\right)^{2}$. For the comparison of data to BFKL calculations the following quantity, 
which is a measure of the length of the gluon ladder, is defined:

$$
Y \equiv \ln \left(s_{\mathrm{ee}} y_{1} y_{2} / \sqrt{Q_{1}^{2} Q_{2}^{2}}\right) \simeq \ln \left(W^{2} / \sqrt{Q_{1}^{2} Q_{2}^{2}}\right) \equiv \bar{Y}
$$

where the approximation requires $W^{2} \gg Q_{i}^{2}$.

\section{Event selection}

The data samples used in the analyses correspond to integrated luminosities of $592.9 \mathrm{pb}^{-1}$ (OPAL), $617 \mathrm{pb}^{-1}$ (L3) and $640 \mathrm{pb}^{-1}$ (ALEPH) collected in the years 1998-2000 at $e^{+} e^{-}$centre-of-mass energies $\sqrt{s_{e e}}=189-209 \mathrm{GeV}$. Double-tagged two-photon events were selected with the following set of cuts (for detailed values see Table 1): a) two electrons with sufficient energies, $E_{i}$, and polar angles, $\theta_{i}$, in

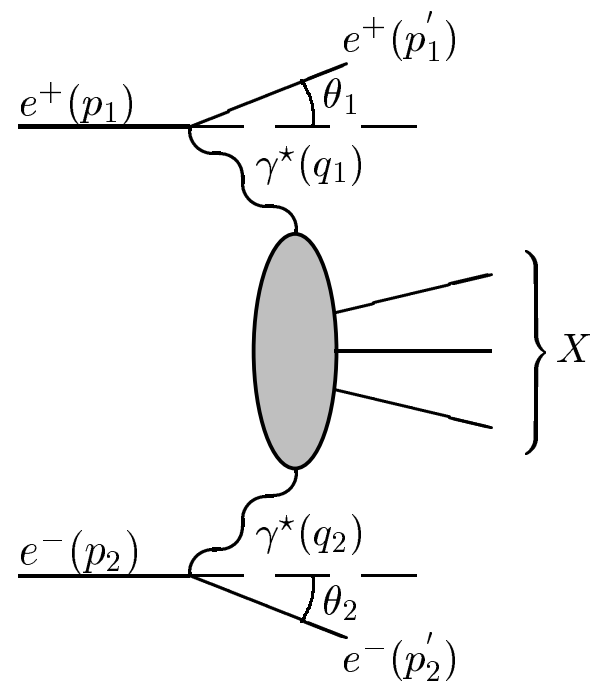

Figure 1: The diagram corresponding to the process $\mathrm{e}^{+} \mathrm{e}^{-} \rightarrow \mathrm{e}^{+} \mathrm{e}^{-}$hadrons. the range covered by small angle electromagnetic calorimeters, should be observed, b) the visible invariant mass, $W_{\text {vis }}$, of the event should be sufficiently large, c) at least $N_{\text {ch }}$ tracks or $N_{\text {part }}$ particles have to be found, d) there should be no additional (i.e. apart of the scattered electrons) single object with an energy above $E_{\max }$, e) events should have a well defined vertex in both $z$ direction $\left(z_{0}\right)$ and in the plane perpendicular to that direction $\left.\left(d_{0}\right), \mathrm{f}\right)$ missing momentum in $z$ direction, $\sum p_{z}$, should be small, g) remaining Bhabha events with random overlap of hadronic activity are tagged using the back-to-back topology of the scattered electrons. A cut against offmomentum background on the total visible energy, $\sum E$, is also applied. With these cuts 175 (OPAL), 891 (ALEPH), 491 (L3) events are selected in the data. Among these events, we expect 42.4 (OPAL), 206.1 (ALEPH), 87 (L3) background events coming from another $\mathrm{e}^{+} \mathrm{e}^{-}$physics processes $\left(\mathrm{e}^{+} \mathrm{e}^{-} \rightarrow \mathrm{e}^{+} \mathrm{e}^{-} \tau \bar{\tau}(e e)\right.$ and single tagged events with a hadron misidentified as an electron (L3)) or from an overlap of off-momentum electrons with single tagged or untagged two photon events.

A PHOJET (OPAL), PYTHIA (ALEPH) or TWOGAM (L3) Monte Carlo samples are used to correct the data for acceptance and resolution effects. It has been veryfied that the sum of the signal as predicted by above MC models and of the background processes gives a good description of all important kinematical variables.

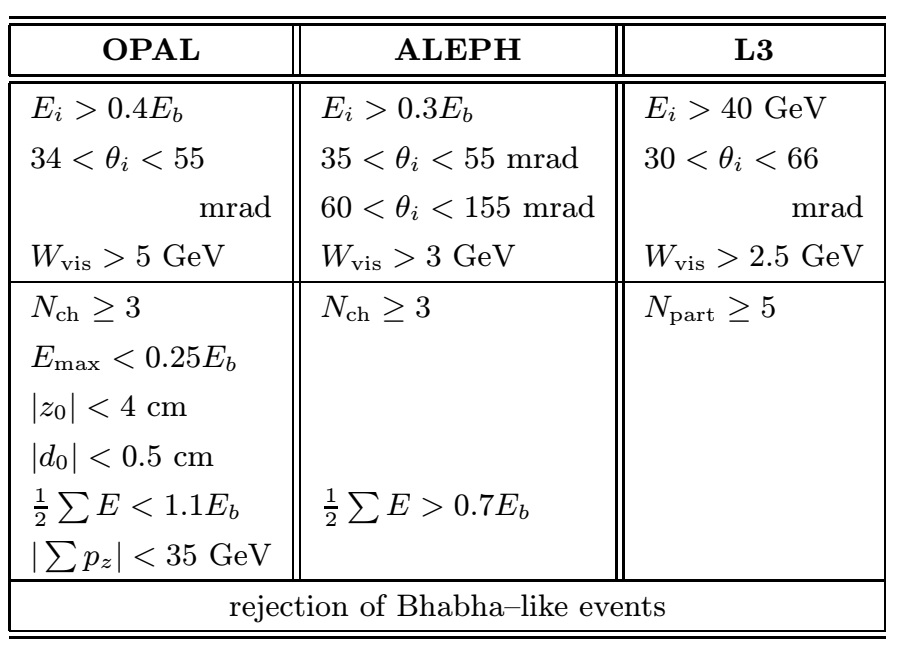

Table 1: Double tag selection cuts used in the analyses. 
QED radiative corrections have been studied by L3 and OPAL using the TWOGAM and BDK programs. We have found that the corrections are very large $(>50 \%$ for high $\bar{Y}$ ) when $W_{\text {vis }}$ is calculated from final state electrons momenta, and negligible when $W_{\text {vis }}$ is calculated from the hadronic final state. The ratios of the radiative to the Born cross section for different subprocesses estimated using TWOGAM are shown in Fig.?.? a a function of $\bar{Y}$. The relative contribution of the subprocesses to the total cross section is shown in Fig.2.27. In the analyses presented here to calculate $\bar{Y}$, ALEPH and OPAL used $W_{\text {vis }}$ obtained
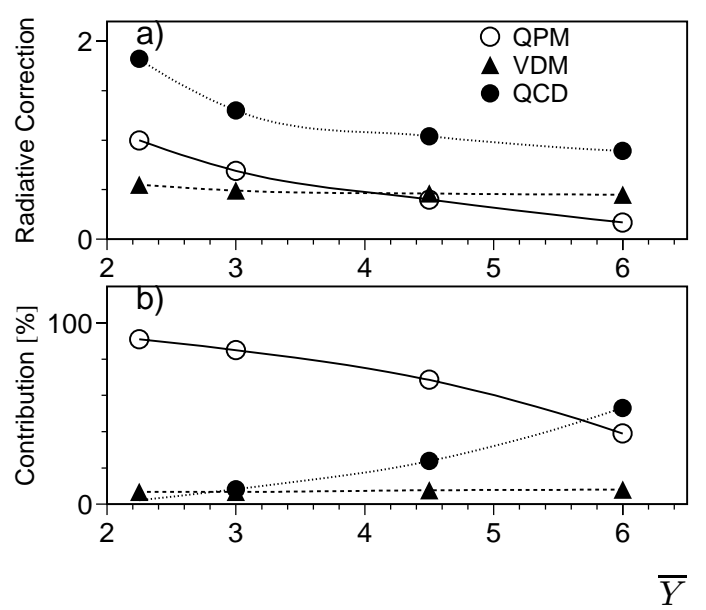

Figure 2: The QED radiative corrections as a function of the variable $\bar{Y}$. from the four-momenta of final state hadrons, and did not apply any correction due to radiative effects to the data, and L3 used $W_{\text {vis }}$ obtained from the four-momenta of the scattered electrons and corrected the measured cross sections for the radiative effects.

\section{Results}

The cross-section for the process $\mathrm{e}^{+} \mathrm{e}^{-} \rightarrow \mathrm{e}^{+} \mathrm{e}^{-}$hadrons has been measured in the kinematic region defined in the Table 2. From the $\mathrm{e}^{+} \mathrm{e}^{-}$cross-section we extract the cross-section for the reaction $\gamma^{*} \gamma^{*} \rightarrow$ hadrons using transverse luminosity function [i]1], $L_{T T}$, calculated separately for each bin using GALUGA. The cross-sections are presented as a function of $x, Q^{2}, W$, the azimuthal correlation between the two electrons $\Delta \phi$ and the variable $\bar{Y}$ in Fig.3-5 for OPAL, in Fig.6 for L3 and in Fig.7 for ALEPH. In Fig.3 $Q^{2}$ refers to the maximum of $Q_{1}^{2}$ and $Q_{2}^{2}$, and $x$ is the corresponding value of $x_{i}$. The total cross-section for the process $\mathrm{e}^{+} \mathrm{e}^{-} \rightarrow \mathrm{e}^{+} \mathrm{e}^{-}$hadrons measured by OPAL in phase space defined in Table 2 , is $0.35 \pm 0.04$ (stat) ${ }_{-0.08}^{+0.04}$ (sys) pb. The expected cross-section from PHOJET is $0.39 \pm 0.02$ (stat) pb, while the prediction for QPM using massive quarks is $0.27 \pm 0.02$ (stat) pb and the NLO predictions for the reaction $e^{+} e^{-} \rightarrow e^{+} e^{-} q \bar{q}$, using massless quarks, is $0.35 \mathrm{pb}$. Both PHOJET1.10 and QPM describe the data equally well for the cross sections in $x, Q^{2}$, $W, \bar{Y}$. PHOJET1.10 does not describe the $\Delta \phi$ distribution whereas QPM reproduces the shape of the distribution. Also the NLO calculation for the reaction $\mathrm{e}^{+} \mathrm{e}^{-} \rightarrow \mathrm{e}^{+} \mathrm{e}^{-} q \bar{q}$ is in accord with the data. The $\gamma^{\star} \gamma^{\star}$ cross section in a function of $Q_{1} Q_{2}$ (Fig.6b) is fitted with the formula $f=A /\left(Q_{1} Q_{2}\right)$, expected by pQCD. The fit reproduces the

\begin{tabular}{|r||c||c|}
\hline \multicolumn{1}{|c||}{ OPAL } & \multicolumn{1}{c|}{ ALEPH } & \multicolumn{1}{c|}{ L3 } \\
\hline \hline$E_{i}>0.4 E_{b}$ & $E_{i}>0.3 E_{b}$ & $E_{i}>40 \mathrm{GeV}$ \\
$34<\theta_{i}<55$ & $35<\theta_{i}<155$ & $30<\theta_{i}<66$ \\
$\mathrm{mrad}$ & $\mathrm{mrad}$ & $\mathrm{mrad}$ \\
$W_{\gamma \gamma}>5 \mathrm{GeV}$ & $W_{\gamma \gamma}>3 \mathrm{GeV}$ & $W_{\gamma \gamma}>5 \mathrm{GeV}$ \\
\hline \hline
\end{tabular}

Table 2: Phase space for the cross section measurements used in different experiments. data well with $A=81.8 \pm 6.4 \mathrm{nb} / \mathrm{GeV}^{2}$, $\chi^{2} /$ d.o.f. $=1.2 / 3$. 

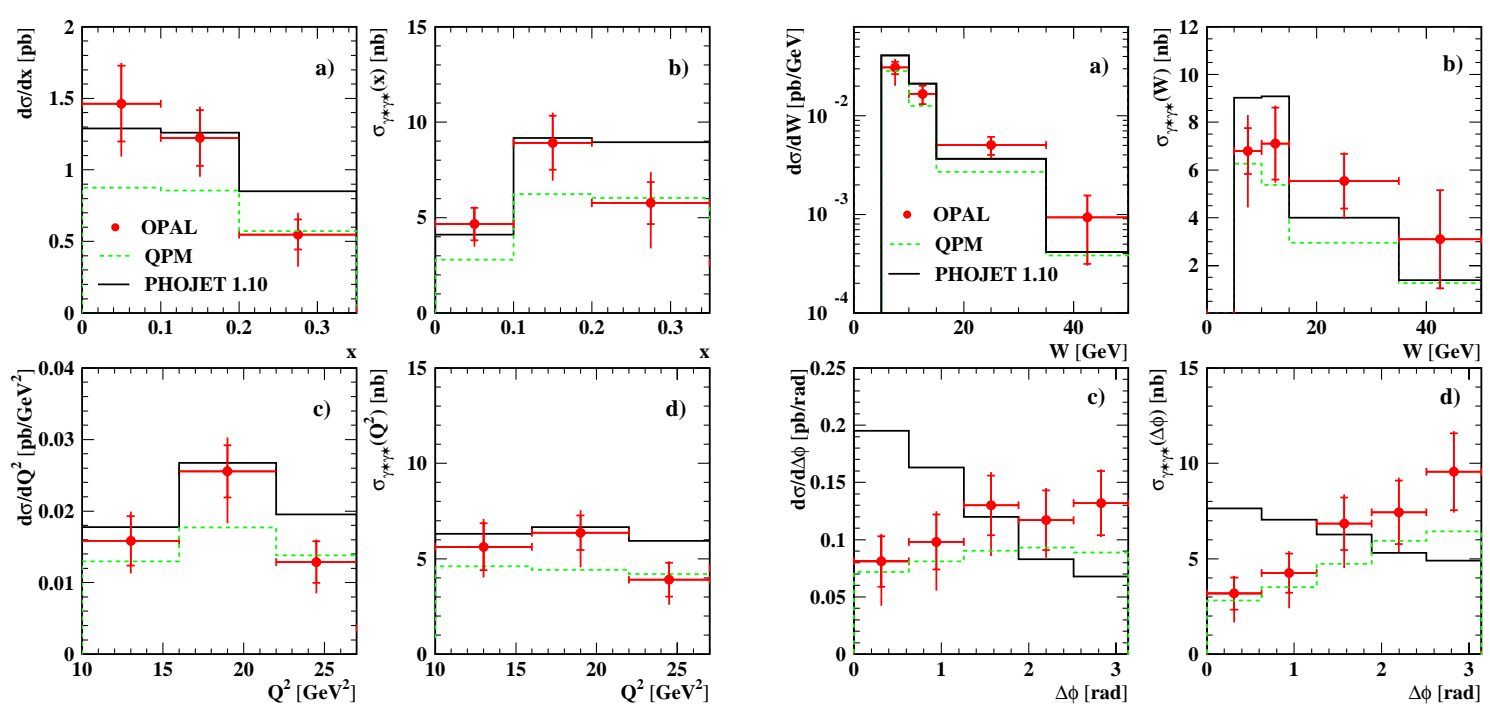

Figure 3: Cross-sections for the process $\mathrm{e}^{+} \mathrm{e}^{-} \rightarrow \mathrm{e}^{+} \mathrm{e}^{-}$hadrons and for the process $\gamma^{*} \gamma^{*} \rightarrow$ hadrons as functions of $x(\mathrm{a}, \mathrm{b})$ and $Q^{2}(\mathrm{c}, \mathrm{d})$. Data are shown as full dots with statistical (inner error bars) and statistical and systematic errors added in quadrature (outer error bars). Predictions of PHOJET1.10 are shown as solid lines, and those of QPM as dashed lines.

Figure 4: As in Fig. 3 but cross-sections are shown as functions of $W(\mathrm{a}, \mathrm{b})$ and $\Delta \phi(\mathrm{c}, \mathrm{d})$.

The data rule out BFKL cross sections which are as large as those predicted by LO-BFKL calculations. NLO-BFKL and calculations including dominant higher order corrections predict smaller effects in the LEP range and are found to be consistent with the measured cross sections. The limited statistics and available $\bar{Y}$ range of the data prevent establishing or ruling out the onset of BFKL dynamics in this reaction.

When transformed to the same phase space using Monte Carlo models the cross sections measured by different experiments are in good agreement beetwen themselves.

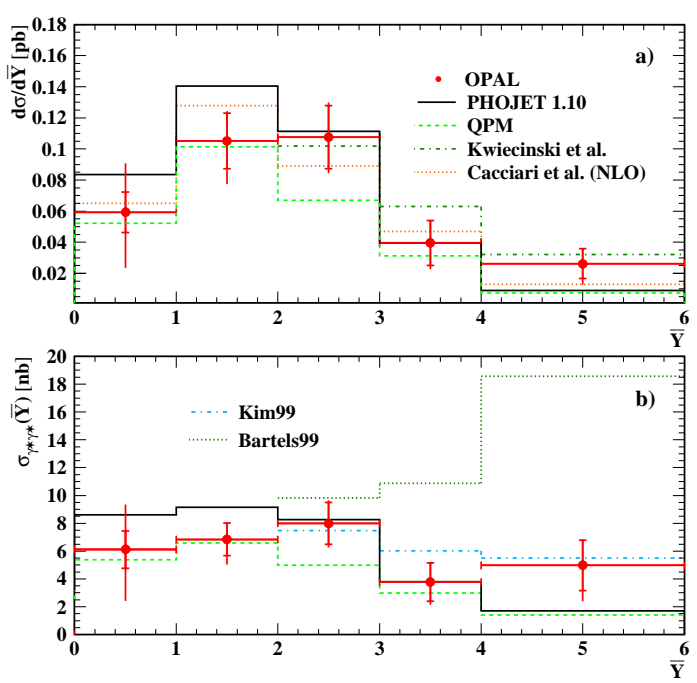

Figure 5: Cross-sections for the process $\mathrm{e}^{+} \mathrm{e}^{-} \rightarrow$ $\mathrm{e}^{+} \mathrm{e}^{-}$hadrons and the process $\gamma^{*} \gamma^{*} \rightarrow$ hadrons for $\left\langle Q^{2}\right\rangle=17.9 \mathrm{GeV}^{2}$ as a function of $\bar{Y}$. Data are shown as full dots with statistical (inner error bars) and statistical and systematic errors added in quadrature (outer error bars). Predictions of PHOJET1.10 are shown as the solid lines, that of NLO calculation of the process $\mathrm{e}^{+} \mathrm{e}^{-} \rightarrow \mathrm{e}^{+} \mathrm{e}^{-} q \bar{q}$ from Cacciari et al. as dotted lines and those of QPM as dashed lines. BFKL calculations shown are: a LO one from Bartels et al. (Bartels99), NLO from Kim et al. (Kim99) and the HO calculation from Kwiecinski et al. 

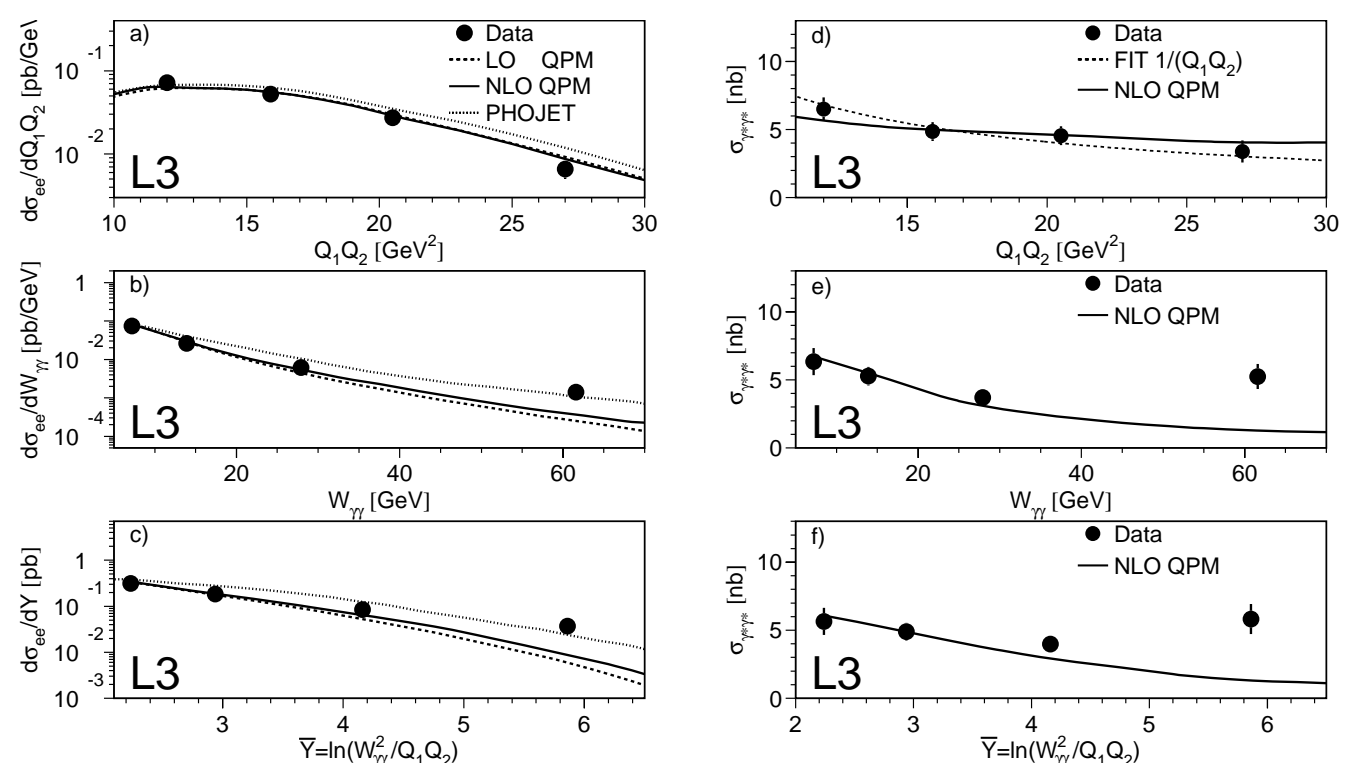

Figure 6: Cross-sections for the process $\mathrm{e}^{+} \mathrm{e}^{-} \rightarrow \mathrm{e}^{+} \mathrm{e}^{-}$hadrons and for the process $\gamma^{*} \gamma^{*} \rightarrow$ hadrons as functions of $Q_{1} Q_{2}(\mathrm{a}, \mathrm{d}), W_{\gamma \gamma}(\mathrm{b}, \mathrm{e})$ and $\bar{Y}(\mathrm{c}, \mathrm{f})$. Data are shown as full dots with statistical and systematic errors added in quadrature. Predictions of PHOJET are shown as dotted lines, those of LO QPM as dashed lines, and NLO QPM as solid lines.
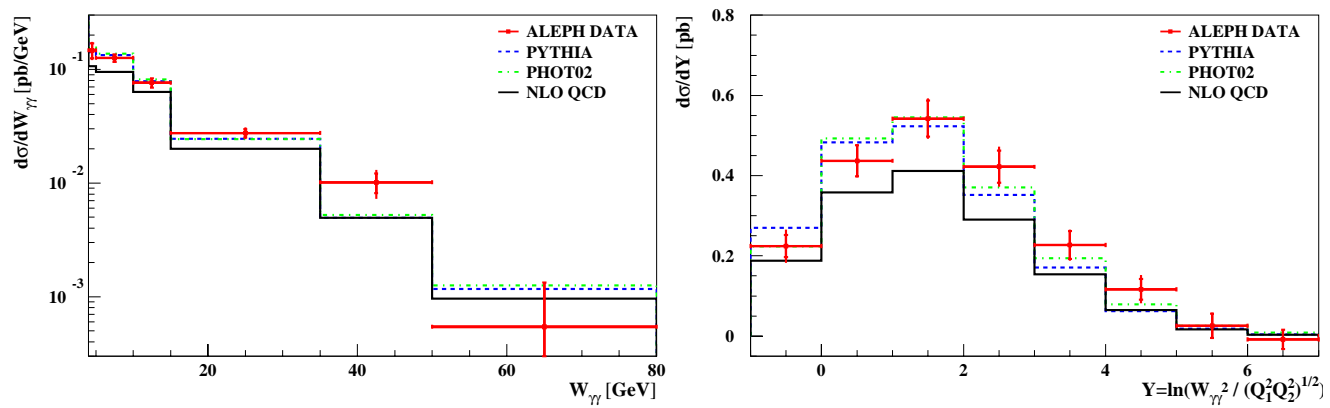

Figure 7: Cross-sections for the process $\mathrm{e}^{+} \mathrm{e}^{-} \rightarrow \mathrm{e}^{+} \mathrm{e}^{-}$hadrons as functions of $W_{\gamma \gamma}$ (left) and $\bar{Y}$ (right). Data are shown as full dots with statistical (inner error bars) and statistical and systematic errors added in quadrature (outer error bars). Predictions of PYTHIA are shown as dashed lines, those of PHOT02 as dashed-dotted lines, and NLO QPM as solid lines.

\section{References}

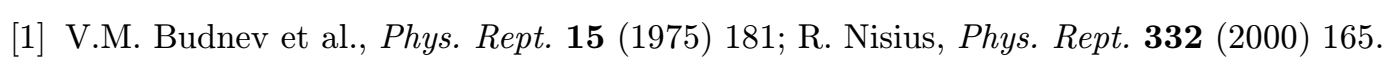

[2] G.Prange, Measurement of the hadronic cross-section of double tagged $\gamma \gamma$ events at ALEPH, Proceedings of the PHOTON 2001 Conf., Ascona, to be published by World Scientific.

[3] C.H.Lin, Double tagged events in two photon collisions at L3 experiment, Proceedings of the PHOTON 2001 Conf., Ascona, Switzerland, to be published by World Scientific.

[4] OPAL Collaboration, G.Abbiendi et al., Measurement of the Hadronic Cross-Section for the Scattering of Two Virtual Photons at LEP, hep-ex/0110006, submitted to Eur. Phys. J. C. 